\title{
Analysis of roundabout capacity under high demand flows
}

\author{
H. M. N. Al-Madani ${ }^{1} \&$ M. Saad ${ }^{2}$ \\ ${ }^{1}$ Department of Civil Engineering \& Architecture, \\ College of Engineering, University of Bahrain, Bahrain \\ ${ }^{2}$ Ministry of Interior, Bahrain
}

\begin{abstract}
Estimation of the entry capacity of roundabouts greatly varies between one method and another in their input requirements, model complexity and estimate accuracy. Several available models for the capacity estimation at approach entries of the roundabouts are tested here and compared for high traffic demand condition. The estimated entry capacities along with the actual entry demand are then compared for various gathered circulating flows. Two models for capacity estimate are developed for triple circulating lanes by triple and dual entry lanes. Thirteen roundabouts in Bahrain are used for the models' development and for the comparison purposes between the various tested methods. The geometric data were gathered from the actual drawings, scaled aerial photographs and from the field. The necessary traffic data were gathered during peak periods. Substantial differences in estimating capacities were observed between the various available international methods. This makes the judgment of accepting or rejecting the estimated capacity difficult. The developed model fall well in between the tested international models and matched the actual data reasonably well. The methods with complicated input parameters and extensive equations, as aaSIDRA, UK RODEL, French and Indian methods, did not prove to be better than much simpler ones like Swiss or FHWA methods. The findings are quite essential for traffic planners in making judicious decisions regarding roundabouts' performance. There is a real need for a more consistent model for the capacity estimation of roundabouts.
\end{abstract}

Keywords: roundabout capacity, maximum entry flow, circulating flow, conflicting lanes, entry lanes, RODEL, aaSIDRA, GIRABASE, NAASRA. 


\section{Background}

Current roundabouts came as a replacement of traffic circles (rotaries) to overcome some of the practical deficiencies associated with circles such as locking up of the traffic and development of long queues in the circles. This is because of the right of way given to the vehicles entering the circles. In order to avoid such blocking queues, the entering traffic should yield, or give way, to circulating ones. This is the main principle of the current roundabouts. Roundabouts are quite popular in erstwhile British Colonial countries. All the traffic circles in Bahrain were converted into conventional roundabouts, soon after Britain, in the sixties. Roundabouts are widely spreading in many other countries because of their advantages over other types of intersection control. The main principle reason was the profit of safety over other cross roads (Guichet [1]). Reduction of $89 \%$ in fatal and incapacitating crashes was observed in the USA after adopting roundabouts in replacement to other types of intersection control [2]. This is due to reduction in approaching speeds, fewer conflict points and no direct left turning. Some of the other advantages include minimum maintenance cost and nice landscape. However, they also carry several serious disadvantages, many of which are usually overlooked. Some are as follows: drivers frustration due to unpredictable long queues during rush hours, when compared with traffic signals (Al-Madani [3]), limitation of bicycle and pedestrian facilities, high construction cost, large land requirements, high tail end accidents, frequent rutting failures at approach entries of flexible pavements, limitation of the technological support or software, compared with traffic signals, and limitation of entry and circulating lanes.

\subsection{History of roundabouts}

Traffic circles have been part of the transportation system in the United States and in some of the European countries long back. They have been used in New York since 1905. High crash experience and congestion in the circles led rotaries fall out of favor in America since the mid-1950. Internationally, the experience with traffic circles was equally negative, with many countries experiencing circles that locked up as traffic volumes increased (Taekratok [4]).

The roundabouts were developed in the United Kingdom. It adopted a mandatory "Give-way" rule at all the circular intersection, which required entering traffic to give way, or yield, to circulating traffic. Wardrop [5], Britain TRL researcher, developed some models related to roundabout capacity in 1957 . The "Give-Way" rule prevented circular intersections from locking up, by not allowing vehicles to enter the intersection until there were sufficient gaps in the circulating traffic. The roundabout represents a substantial improvement, in terms of operations and safety, when compared with older rotaries. Therefore, many countries have adopted them as a common intersection form and some have developed extensive design guides and methods to evaluate the operational performance of modern roundabouts. Al-Madani [3] found that roundabouts perform better than traffic signals when the traffic demand is low. However, as 
the demand increases at a roundabout so does the queue length and the delays. Beside drivers' frustration due to the unpredictable delays at congested roundabouts, when compared with traffic signals, long queues are inevitable. The latter leads to police intervention in order to control the queues and direct the traffic flow. Such phenomena lead Bahrain Government to convert most of the major roundabouts on the main roads into signalized intersections. Some were constructed during the sixties. Akçelik [6] also observed roundabout to perform well at low to medium flow conditions. Clear capacity reduction was observed at high demand levels.

\subsection{Roundabout capacity}

Roundabout capacity is the main determinant parameter for the performance measure of many other parameters as delay and queue length. It is the maximum sustainable flow rate that can be achieved during a specific period under prevailing traffic, geometric and control conditions. Capacity is service rate and is different than maximum volume that an intersection can handle (Akçelik [6]), which is the practical capacity under high demand volume; not under prevailing conditions.

Most of the capacity models are either analytical ones based on gap acceptance, with no actual observations, or empirical regression ones based on observed geometric and flow parameters (Figure 1). Kimber [7] stated that capacity estimates based on gap acceptance models are not suitable for the application in England. This was due to the problems related to human behavior. On the other hand, Fisk [8] finds regression models to be difficult for frequent application due to large number of data requirements.

The maximum flow rate that can be accommodated at a roundabout entry depends mainly on the following factors; the circulating flow on the roundabout that conflicts with the entry flow, exiting flow and the geometric elements of the roundabout. When the circulating flow is low, drivers at the entry are able to enter the roundabout without any significant delay. The larger the gaps, i.e. the headways, in the circulating flow the more useful they are for the drivers entering the roundabout. In fact, more than one vehicle may enter in each gap. As the circulating flow increases, the size of the gaps in the circulating flow decreases, and the rate at which vehicles enter the roundabout decreases. The geometric elements of the roundabout also affect the rate of entry flow. The most important geometric elements are the width of the entry, the width of the circulatory roadway, or number of lanes around the central island. Two entry lanes permit nearly twice the rate of entry flow as does one lane. Wider circulatory roadways allow vehicles to travel alongside, or follow, each other in tighter bunches and so provide longer gaps between bunches of vehicles. The flare length also affects the capacity. The inscribed circle diameter and the entry angle have minor effects on capacity. In fact, TRL invented mini roundabouts which performed better than some larger ones in terms of capacities (Kimber [9]).

There are several analytical and empirical models for the capacity estimations. Some are very well known and others are less popular. Many 
countries utilize models developed by their own researchers as to meet their needs. However, capacities estimated through these models widely differ between one model and another. Some are very simple and require minimum data entry as the Swiss, the US Highway Capacity Manual (HCM) and Federal Highway Administration (FHWA) models, others are complicated and require extensive data entries as UK RODEL, Australia aaSIDRA, French GIRABASE, and Indian models. The UK RODEL and the Australian aaSIDRA models are the most famous ones. The question of how good the capacity estimate of each model is, requires further investigations. The need for capacity evaluation developed through the various models and software programs, available worldwide, was stressed by Jacquemart [10].

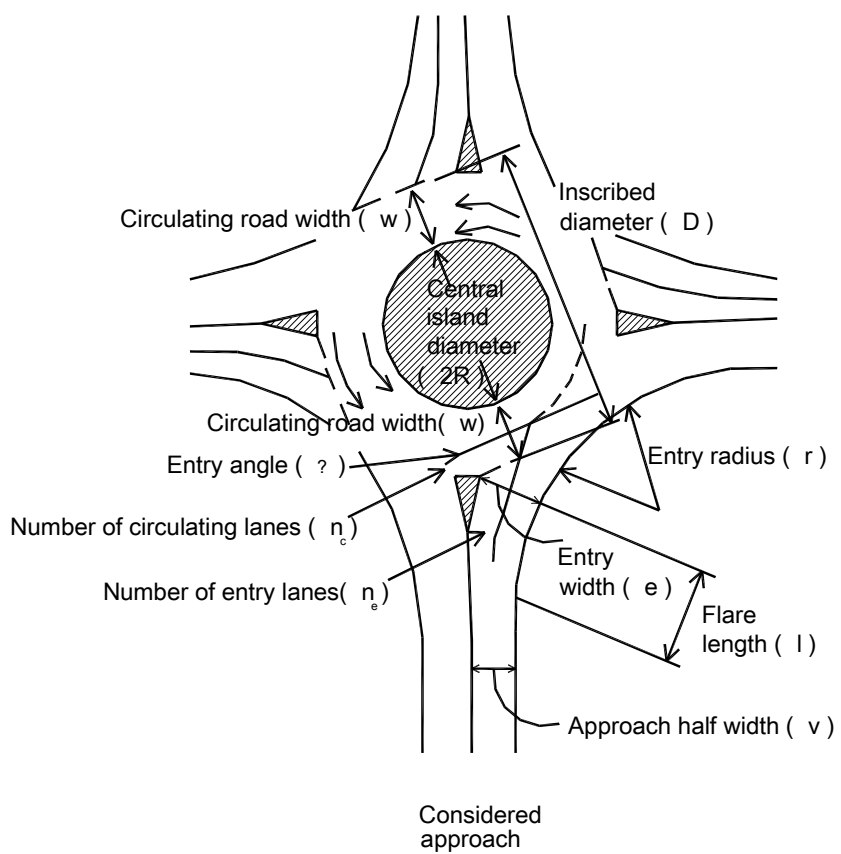

Figure 1: Geometric parameters needed for the roundabout capacity models.

\subsection{International roundabout capacity models}

Most of the capacity models are developed in the West European countries and in Australia. There are several models currently used by both the researchers and the operators. Some are widely used others are not. Stanek and Milan [11] stated that most of the known capacity models can be calibrated by modifying the intercept values, as in RODEL model, and follow up headway, as in aaSIDRA model. The UK model (RODEL) is based on the work carried out by Kimber and Hollis [12] and Kimber [9] for TRL. This method has been incorporated into a software packages widely known as RODEL (Crown [13]). The Australian model- aaSIDRA capacity expressions is published in Australia by 
Akçelik et al. [14, 15], and have been incorporated into a widely known software called aaSIDRA. Many parameters are employed in aaSIDRA (Akçelik and Associates [6]) for sensitivity analysis to count for driver behavior. The Australian model- NAASRA is developed by National Association of Australian State Road Authorities [16,17] and Adams et al. [18]. The German model is derived from the Tanner-Wu capacity equation and has been introduced officially into the German Highway Capacity Manual in 2001 [19] (Brilon et al. [20]). The detailed formula for entry flow is discussed by Wu $[21,22]$. The French Model is based on the work carried out by Louah [23] which was later incorporated into a model known as GIRABASE [24]. The Swiss Capacity Model includes the influence of exiting flow and the width of the splitter island which are not utilized in most of the other models. There are two methods currently found in the US literature. The first is found in the Highway Capacity Manual- HCM (Transportation Research Board [25]). The second is a simplified British linear regression method cited in the Federal Highway Administration (FHWA) Roundabout Guidebook [26]. The basic concept of the Indian Model is based on Wardrop late fifties' literatures. The modified equations are as follows (Kadiyalli [27]).

\section{Objectives}

Two main objectives are researched here. The first is to develop capacity models for roundabouts with dual and triple entry lanes under saturated traffic demands. The models estimate the maximum entry flow given the circulating flows for given set of geometric conditions. The second is to make a comparison between the various available international roundabout capacity models. The developed models are compared with nine international ones. These include the following: UK (RODEL), Australian aaSIDRA and NAASRA, German, French (GIRABASE), Swiss, US FHWA and HCM, and Indian models.

\section{Methodology}

The capacity models are developed using 13 major roundabouts, out of 15 selected ones, in Bahrain. These cover either two or three entry and circulating lanes. The models are developed based on the maximum entry flow, i.e. capacity, and the corresponding circulating flow. The former will be used as the dependent variable while the latter as independent one. The data are collected during the Peak hours to ensure higher saturation flows necessary for the development of capacity models. Furthermore, they provide better basis for comparison (Seiberlich, [28]) with other models. Least square regression models are used to develop the models. The data will be regressed based on linear, logarithmic, polynomial, power exponential methods. The models with best fit in terms of higher $\mathrm{R}^{2}$ values will be selected to represent the data. One model will be selected to represent roundabouts with three entry lanes by three conflicting lanes another model will be selected to represent two entry lanes by three conflicting lanes' roundabouts. 
All the necessary traffic and geometric data are carefully gathered and properly considered to avoid misinterpretations of the parameters, used in the various models, as they may vary from one method to another. These parameters are unified and given the same abbreviated letters as used here. Afterward, the roundabouts' maximum entry flows are determined for each approach of the selected roundabouts. These are determined using the models discussed earlier using advanced EXCEL features. The estimated maximum entry flows are based on the equations stated earlier. None were produced through the software corresponding to the above methods. This is to avoid unrealistic assumptions and default values utilized in the software for simplification purposes. The maximum entry capacity is analyzed per approach entry flow, not per individual lane, since most of the considered models utilize the traffic per approach entry. This is just not to add further complications to the models which are already complex. Furthermore, researchers prefer them over individual lane determination (Seiberlich [28]). Each of the calculated capacity is first compared with the actual demand, and then all the determined capacities are compared against their corresponding circulating flows.

\section{Data gathering}

Fifteen roundabouts were first selected across Bahrain for the investigation. The selected roundabouts carried saturated traffic flow during peak periods, relatively large inscribed diameters, i.e. over $60 \mathrm{~m}$, and similar approaching lane widths. The above were short listed to thirteen roundabouts based on the following requirements: multi-entry lanes, multi-circulating lanes, either four or five approaching legs, being on the main roads, and minimal approaching grades. These are meant to lead to better consistency in the results and fair comparison between the considered models. The geometric parameters require careful attention and high caliber to measure. Many are very tedious to acquire. The process of gathering the geometric parameters was not an easy one. The geometric parameters of the roundabouts were gathered from actual drawings of the roundabouts, GIS maps with scale of (1:2000), scaled aerial photos, and actual field measurements. These were necessary to cross-check the data extracted from one source to another, to measure the missing geometric parameters from the original drawings and to compare the proposed drawings with the actual ones. The traffic flow counts during both morning and evening peaks were gathered for each approach.

\section{Developed models}

Various types of regression models are tested here. The models best fit the gathered maximum entry flow versus circulating flow data along with their corresponding $\mathrm{R}^{2}$ values are presented in Table1. These covered the following four main types: linear, logarithmic, exponential and power regression models. The exponential model fits the tested data, for the case of three entry lanes by three circulating lanes, best when compared with the other models because it 
holds the highest $\mathrm{R}^{2}$ value (0.56). However, the linear relationship does not differ much from the exponential model one in terms of $\mathrm{R}^{2}$. The models though being insufficiently high, they looks fine for such dispersive nature of data. However, the logarithmic model fits the data for the two entry lanes by three circulating lanes reasonably well. The model carried $\mathrm{R}^{2}$ value of 0.81 .

Table 1: $\quad$ Developed models for roundabout capacity.

\begin{tabular}{|l|l|l|l|l|}
\hline \multirow{2}{*}{ Model type } & \multicolumn{2}{|l|}{ Entry lanes=3; Entry lanes=3 } & \multicolumn{2}{l|}{$\begin{array}{l}\text { Entry lanes=2; Entry } \\
\text { lanes=3 }\end{array}$} \\
\cline { 2 - 5 } & $\begin{array}{l}\text { Models } \\
\text { Developed }\end{array}$ & $\mathrm{R}^{2}$ & $\begin{array}{l}\text { Models } \\
\text { Developed }\end{array}$ & $\mathrm{R}^{2}$ \\
\hline Linear & $\begin{array}{l}20607- \\
0.8179 Q_{c}\end{array}$ & 0.504 & $1686-0.429 Q_{c}$ & 0.642 \\
& $\begin{array}{l}6313.9-691 l n \\
Q_{c}\end{array}$ & 0.434 & $\begin{array}{l}58411.4- \\
680.8 \ln Q_{c}\end{array}$ & 0.810 \\
\hline Logarithmic & $2952.9 e^{-0.0007 Q c}$ & 0.562 & $2540.0 e^{-0.0007} Q_{c}$ & 0.609 \\
\hline Exponential & $46855 Q_{c}^{-0.5268}$ & 0.415 & $475961 Q_{c}^{-0.9121}$ & 0.474 \\
\hline Power & & & & \\
\hline
\end{tabular}

The maximum entry flow rates predicted from the selected two models are compared with other international models. The following can be observed when the 3 entry lanes by 3 circulating lanes' model is considered (Figure 2): 1 . The developed model fall well in between the tested international methods. 2. It also fairly matches the NAASRA model at low circulating flow rates but clearly falls above it at medium and high circulating flow rates. 3 . The UK RODEL model clearly showed higher maximum entry flows at medium to high circulating flows when compared with the developed exponential model. The two matched quite well at low circulating flows. 4. The German model matched the developed model fairly well at medium to high circulating flows. At low flow rates the German model showed lower values. 5. The aaSIDRA model falls far above the developed models. 6. The Swiss model falls slightly above the developed model at all the circulating flow rates but the very high values. 6 . The French model falls above the developed model and, in fact, above all the actual values. 7. US HCM model closely matches the developed model. However, it shows slightly higher values at low circulating flows and slightly below it at high rates. The two models match each other fairly well in between low and high values.

The following can observed when considering the maximum entry flow rate for the model developed considering 2 entry lanes by 3 circulating lanes (Figure 2): 1. As in the earlier case, the developed model falls in between the international models as a whole. The model matched the actual data very well. 2 . The model follows closely the German and the US HCM models at all circulating flow rates except at very low flow rates. 3. The US FHWA and the Swiss liner models fall above the developed models at medium to high circulating flow values and below it elsewhere. 4. The UK RODEL model though showing slightly higher values at medium circulating values than the developed model, it matches the model reasonably well at low and high 
circulating values. 5 . The aaSIDRA and the French models fall clearly above the developed model.

\section{Comparison between the international models}

The results for the various roundabout capacity methods versus circulating flows, for the different roundabouts as shown in Figs.2 and 3, clearly show the Indian method to be highly overestimating the capacities for given circulating flows and corresponding geometric characteristics when compared with the actual demand and with those estimated from other tested methods. Furthermore, the circulating flows versus the estimated capacities for the various methods, excluding the Indian method since they clearly fall out of the range of the other methods, along with the actual demand show the following conclusions:

1. The results indicated wide variations in capacity estimates of roundabouts among the various international methods. Some showed to be more reasonable than others when compared with actual demand during rush hours. Such differences confuse the practicing engineers and consultants. The actual entry demand was found to be higher than that estimated through the Australian NAASRA method for both dual and triple entry lanes' roundabouts. In other words the method under estimates the capacity estimates. This is especially true for medium and high circulating flow conditions.

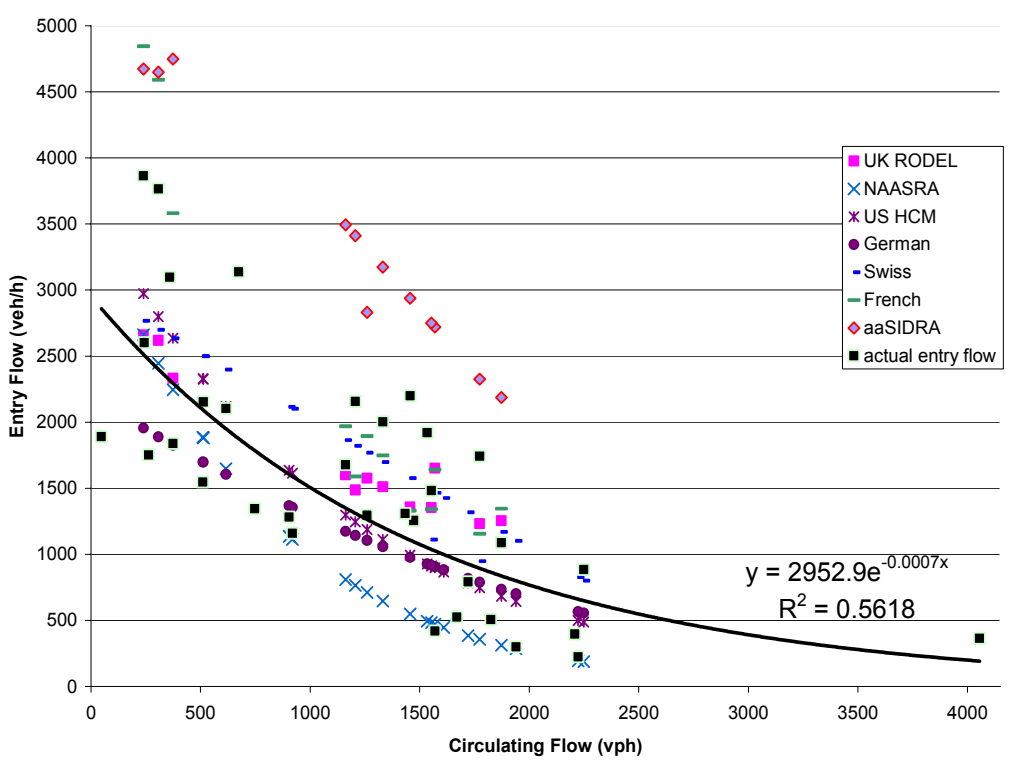

Figure 2: $\quad$ Developed roundabout capacity model for 3 entry lanes by 3 circulating lanes along with international values. 


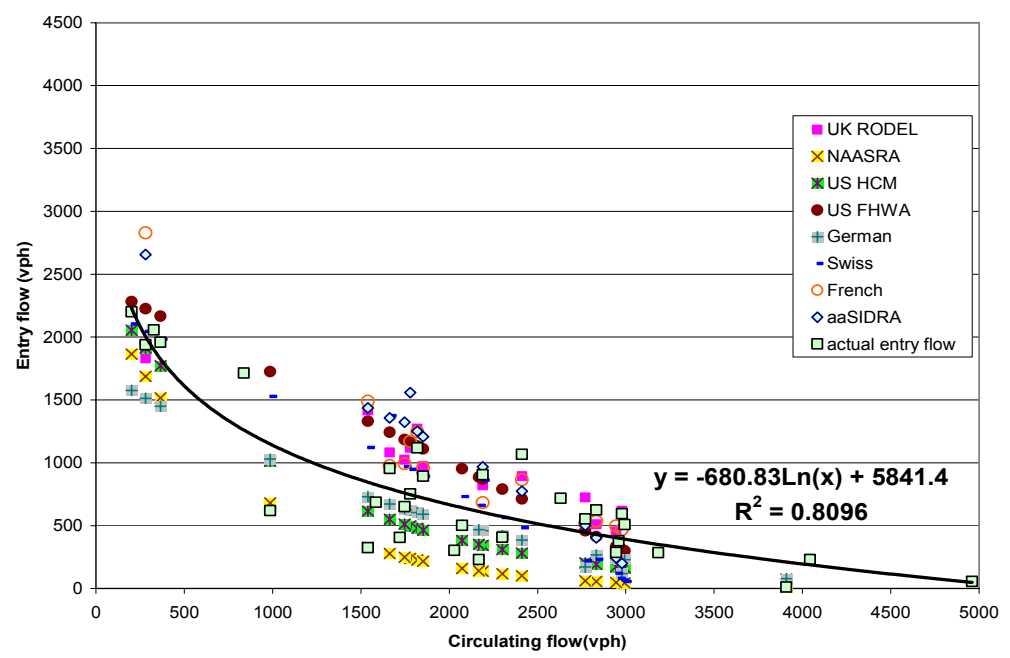

Figure 3: Developed roundabout capacity model for 2 entry lanes by 3 circulating lanes.

While the Australian NAASRA method showed the lowest capacity estimates compared with other international methods and compared with the actual data; Australian aaSIDRA method showed the highest capacity estimates, excluding the Indian method, for circulating flows up to $2700 \mathrm{veh} / \mathrm{h}$. In other words, the maximum entry flows estimated by the two methods showed two extreme sides of predictions. One on the far high side and the other on the low side.

2. Non realistic high capacity estimates are observed for low values of circulating flows when either the Australian aaSIDRA or French methods are considered. This is probably because of the involvement of exponential parameter in the models. The latter may require careful calibration and threshold limits for low circulating flows.

3. Similar to the two Australian methods, i.e. NAASRA and aaSIDRA, the two US methods FHWA and HCM, also showed a high and a low estimate of maximum entry flows, respectively, for the various given parameters when compared with the actual data. In general, the estimated entry flows by the both methods fall between the envelopes of the two Australian methods; especially when the circulating flows falls below $2700 \mathrm{veh} / \mathrm{h}$. The FHWA method is not applicable for triple entry lanes by triple circulating lanes since it is limited to only single or dual lanes.

4. The estimated roundabout entry capacity through the US HCM method showed to be closely matching that determined through the German method. Both tend to show low capacity estimates for given circulating flows, but higher than that estimated using NAASRA method, when compared with UK RODEL, US FHWA, French and aaSIDRA methods.

5. The entry capacities estimated through the UK RODEL and Swiss methods showed quite reasonable match, though the latter is far less complicated than 
the former. Generally, both followed the actual demand quite well, for roundabouts with triple entry lanes by triple circulating lanes, compared with the other methods. Generally, the Swiss method showed slightly higher values than the UK method. The opposite was true for roundabouts with double entry lanes by triple circulating lanes. It is quite interesting to mention that the Swiss method is among the limited methods which utilize the exit flows, beside the circulating flow along with few geometric parameters, to estimate the maximum entry flow.

6. The maximums entry flows estimated through the UK RODEL method also closely match that estimated through the French method as the circulating flows exceed $1000 \mathrm{veh} / \mathrm{h}$.

7. The complicated methods, i.e. those requiring many parameters and involving several equations, as aaSIDRA, UK RODEL, French GIRABASE, and Indian methods, did not show better capacity estimates than much simpler ones, as the Swiss or the US HCM methods, when compared with the actual demands during peak periods. The latter are matching the demand flow reasonably well.

\section{Conclusions and recommendations}

Two models for the capacity estimates of roundabouts are developed here. The first is an exponential model for triple entry and circulating lanes. The second is a logarithmic one for dual entry lanes by triple circulating lanes. Both models matched the actual demand during the peak periods reasonably well and fall in between the international models.

Substantial differences in the capacity estimations were observed among the various available international methods. Such differences make the judgment of accepting or rejecting the estimated capacities very difficult. They also cause confusion to practicing engineers. While the capacities estimated through the Australian NAASRA method showed to be the lowest among those tested, that determined through the Australian aaSIDRA method showed to be among the highest. The US FHWA and HCM also showed a high and a low estimated entry flows, respectively, compared with the actual data and with the other tested methods. The aaSIDRA and the French methods showed nonrealistic high capacity estimates at low corresponding circulating flows. The estimated capacities through the HCM method matched that determined through the German method closely. However, they tend to show low estimated values when compared with the actual data and with the other methods. The UK RODEL and the Swiss methods showed quite reasonable match, though the latter is far less complicated than the former. Both followed the actual demand quite well when compared with the other methods. The UK method also closely matched the French method for moderate to high circulating flows. The complicated methods, those involving many parameters' measurements and extensive calculations, as the aaSIDRA, the RODEL, the French, and the Indian methods did not necessarily showed better capacity estimations than those much simpler ones as the Swiss or the FHWA methods. 
The findings are quite essential for the traffic planners in making judicious decisions regarding roundabouts' performance. There is a real need for a more consistent model for the capacity estimation of roundabouts. Such a model should utilize parameters which can easily be measured and comprehended by the users.

\section{Acknowledgements}

The author wishes to thank Dr. Y. Najjar, Prof. S. Alvi, Mrs. Z. Saleh, Mr. A. Jaafar, H. Qadhi for their assistance during the study.

\section{References}

[1] Guicheet, B. Evolution of roundabouts in France. National Roundabout Conference, TRB, Colorado, USA, 2005.

[2] www.highwaysaefty.org (as on June 2008).

[3] Al-Madani H.M.N., 2003. Dynamic vehicular delay comparison between a police-controlled roundabout and a traffic signal. Transportation Research, 37A, 681- 688 .

[4] Taekroutok, T., 1998. Modern roundabouts for Oregon. Department of Transportation, Research Unit, OR98-SRS22, Salem, USA.

[5] Wardrop, J. G., 1957, The traffic capacity of weaving sections of roundabouts. Proceedings of the First International Conference on Operational research, English Universities Press, London, UK.

[6] Akçelik and Associates, 2005. aaSIDRA In put Guide. Revised version. Akçelik and Associates Pty Ltd, Greythorn, Melbourne, Australia.

[7] Kimber, R. M., 1989. Gap acceptance and empiricism in capacity prediction. Transportation Science, 23 (2), 100- 111.

[8] Fisk, C. S., 1991. Traffic performance analysis at roundabouts. Transportation Research, 25B, 89-102.

[9] Kimber, R. M., 1980. The traffic capacity of roundabouts. TRRL, LR942, Crowthorne, UK.

[10] Jacquemart, G., 1998. Modern Roundabout Practice in the United States. Synthesis of Highway Practice 264, Transportation Research Board, Washington, D.C., USA.

[11] Stanek, D and Milam, R. T. High-Capacity roundabout intersection analysis: going around in circles. National Roundabout Conference, TRB, Colorado, USA, 2005.

[12] Kimber, R. M. and Hollis, M., 1979. Traffic queues and delays at road junctions. Transport and Road Research Laboratory, LR909, Crowthorne, Berkshire, UK.

[13] Crown, R.B., 1987. RODEL1: Interactive Roundabout Design (Software Manual). RODEL Software Ltd. and Council Stoke-on-Trent.

[14] Akçelik, R., Chung, E. and Baseley, M., 1996. Performance of roundabouts under heavy demand conditions. Road and Transport Research, 5(2), 3650 , Victoria, Australia. 
[15] Akçelik, R., Chung, E. and Baseley, M., 1998. Roundabouts: capacity and performance analysis. Research Report APR 321, ARRB, Transport Research Ltd., Vermon South, Australia.

[16] National Association of Australian State Road Authorities (NAASRA), 1998. Guide to Traffic Engineering Practice- Roundabouts. Part 6, APG11.6, National Association of Australian State Road Authorities, Sydney, Australia.

[17] National Association of Australian State Road Authorities (NAASRA),1998. Guide to Traffic Engineering Practice- Intersections at Grade. Part 5, National Association of Australian State Road Authorities, Sydney, Australia.

[18] Adams, M., Barker, D., Rye, T., 2004. Roundabouts. Unit 9, BE71008: Highway Planning and Design. Third Edition, Napier University, School of the Built Environment, Scotland, UK.

[19] German Highway Capacity Manual (Forschungsgesellschaft fur StraBenund Verkehrswesen, Handbuch fur die Bemessung von StraBenverkehrsanlagen, HBS), 2001. FGSV, no. 299, Verlag GmbH, Koln, Germany.

[20] Brilon, W., Wu, N. and Bondzio, L., 1997. Unsignalized Intersections in Germany- A State of the Art 1997. In Proceedings of the Third International Symposium on Intersections Without Traffic Signals (M. Kyte ed.), University of Idaho, Portland, Oregon, USA.

[21] Wu, N., 1997. Capacity of shared- short lanes at unsignalized intersections. In Proceedings of the Third International Symposium on Intersections Without Traffic Signals (M. Kyte ed.), University of Idaho, Portland, Oregon, USA.

[22] $\mathrm{Wu}, \mathrm{N} ., \mathrm{A}, 2001$. Universal procedure for capacity determination at unsignalized (Priority-Controlled) intersections. Transportation Research B, 35,3 .

[23] Lough, G., 1992. Panaroma critique des models Francais de capacite des carrefours giratoires. Proceedings of the seminar Roundabouts 92, SETRA, BP 100, F 92223 Bagneux, Nantes, France.

[24] GIRABASE. Calculation of roundabout capacity. www.certu.fr (as on June 2008).

[25] Transportation Research Board, Highway Capacity Manual, 2000. National Research Council, TRB, SR 209, Washington D.C.

[26] Federal Highway Administration (FHWA), 2000. Roundabouts: An Information Guide. FHWA- RD-00-067, US Department of Transport, Virginia, USA.

[27] Kadiyalli, L. R., 1999. Traffic Engineering and Transport Planning. Khanna Publisher, New Delhi, India.

[28] Seiberlich, E. L., 2001. A Formulation to Evaluate Capacity and Delay of Multilane Roundabouts in the United States for Implementation into a Trend forecasting Model. M. Sc. Thesis, The University of Wisconsin, Milwaukee, USA. 